\title{
Computed Tomography-guided 125I Radioactive Seed Implantation Therapy for Pancreatic Cancer Pain
}

\author{
Tao Fan and Ju-Ying Zhou \\ Department of Radiotherapy, The First Affiliated Hospital of Soochow University, Suzhou, Jiangsu Province, China
}

\begin{abstract}
Objective: This study aimed to evaluate the short-term curative effect of computed tomography (CT) guided radioactive 125I (125lodine) seed implantation (CTRISI) therapy for advanced pancreatic cancer patients pain.

Study Design: Interventional study.

Place and Duration of Study: Imaging Department and Oncology Department, First Affiliated Hospital of Zhengzhou University, China, from January 2015 to March 2017.

Methodology: A total of thirty-seven patients with advanced pancreatic cancer pain who underwent CTRISI were enrolled. Exclusion criteria were coagulation disorder, multi-organ failure, co-existent pancreatitis and/or uncontrolled hyperglycemia. Cancer pain levels and daily hydroxycodone dose were compared before and after CTRISI, using the Numerical Rating Scale (NRS). Complication after procedure were also noted.

Results: All patients were successfully implanted with 125I seeds via CT. After 1-week, 1-month and 2-month of implantation, the NRS scores were significantly lower than preoperative scores $(p<0.05)$. The daily dose of hydroxycodone in one week and one month after operation were significantly lower than preoperation $(p<0.001)$. However, after 2 -month of implantation, the daily hydroxycodone dose was not different from the preoperative dose $(p=0.198)$. No patient experienced serious complications.

Conclusion: CTRISI could relieve pain effectively in the patients and suppress local tumor progress. This technique helps to relieve the pain symptoms, reduce the amount of painkillers, and improve the quality of life of patients.
\end{abstract}

Key Words: Pancreatic cancer, 125I, radioactive seed implantation, pain, hydroxycodone.

How to cite this article: Fan T, Zhou JY. Computed Tomography-guided 125I Radioactive Seed Implantation Therapy for Pancreatic Cancer Pain. J Coll Physicians Surg Pak 2020; 30(04):364-368. DOI: https://doi.org/10.29271/jcpsp.2020.04.364.

\section{INTRODUCTION}

Nowadays, pancreatic cancer is one of the most common malignancies in the world, with high mortality rates. ${ }^{.}$Surgical resection is the only curative treatment modality for patients with localized pancreatic cancer. ${ }^{2}$ However, more than $80 \%$ of patients present with unresectable locally advanced or metastatic tumors at the time of diagnosis. ${ }^{3,4}$ Systemic chemotherapy is the treatment of choice for unresectable pancreatic cancer to improve the survival, nevertheless most pancreatic cancers are refractory to chemo-therapy. ${ }^{5}$

In pancreatic cancer, particularly in pancreatic ductal adenocarcinoma (PDAC), perineural invasion (PNI) has a prevalence between 70 and $100 \%$, surpassing any other solid tumor. ${ }^{6}$ Tumor cells can not only directly infiltrate the nerve to cause pain, but also can act on the myelin sheath of the nerve, increase the effect of pain conduction pathway, and aggravate the pain. ${ }^{7}$

Correspondence to: Prof. Ju-ying Zhou, Department of Radiotherapy, The First Affiliated Hospital of Soochow University, 188 Shizi street, Gusu District, Suzhou, Jiangsu Province, 215006, China

E-mail: zhoujuying2017@163.com

Received: September 05, 2019; Revised: February 16, 2020; Accepted: April 08, 2020

DOI: https://doi.org/10.29271/jcpsp.2020.04.364
Cancer pain seriously affects the quality of life of patients and aggravates the progression of the disease. In addition, as pain is major threat to quality of life, the relief of pain has emerged as a treatment principle in the advanced cancer. Therefore, early effective analgesia and improving the quality of life of patients have become an important part of comprehensive treatment of advanced pancreatic cancer.

The objective of this study was to investigate the clinical efficacy of CT guided 125-lodine seed implantation (CTRISI) for advanced pancreatic cancer pain, in order to provide important reference for CTRISI applied in the field of advanced pancreatic cancer pain.

\section{METHODOLOGY}

This study was conducted at the Imaging and Oncology Departments, First Affiliated Hospital of Zhengzhou University, China, from January 2015 to March 2017 and was approved by the Hospital Ethical and Research Committee.

A total of 45 patients with advanced pancreatic cancer pain were referred for CTRISI. However, 4 patients did not undergo surgery: two patients each had either severe respiratory infection or could not stay in supine position. Four patients were lost to follow up, and these patients either had data for one week or no data for two months. Finally, 37 patients were included for analysis.

Inclusion criteria were pathological diagnosis with pancreatic cancer pain, unresectable pancreatic cancer, expected survival time greater than three months, non-tolerant to chemotherapy and refused external radiotherapy. 
Table I: Intensity of pain before and after treatment [n (\%)].

\begin{tabular}{|l|c|c|c|}
\hline Groups & No Pain & Mild Pain & Moderate Pain \\
\hline Preimplantation & $0(0.0)$ & $12(32.4)$ & $24(64.9)$ \\
\hline Post 1-week & $1(2.7)$ & $34(91.9)$ & $2(5.4)$ \\
\hline Post 1-month & $2(5.4)$ & $30(81.1)$ & $5(13.5)$ \\
\hline Post 2-month & $0(0.0)$ & $21(56.8)$ & $16(43.2)$ \\
\hline
\end{tabular}

Table II: Within-group and pairwise comparisons of NRS score before and after CTRISI.

\begin{tabular}{|l|c|c|c|}
\hline Groups & NRS score & $\begin{array}{c}\text { F } \\
\text { p-value }\end{array}$ & 95\% $\mathbf{C l}$ \\
\hline Preimplantation & $4.0 \pm 1.6$ & $\begin{array}{l}357.62 \\
<0.001\end{array}$ & \\
\hline Post 1-week & $2.5 \pm 0.9$ & & $<0.001$ \\
\hline Post 1-month & $2.5 \pm 1.0$ & $<0.001$ & 0.005 \\
\hline Post 2-month & $3.4 \pm 0.9$ & & $0.146-1.098$ \\
\hline Cl: Confidence Interval & & & 0.104 \\
\hline
\end{tabular}

Table III: The pairwise comparisons of daily hydroxycodone dose before and after CTRISI.

\begin{tabular}{|c|c|c|c|c|}
\hline Groups & Daily hydroxycodone dose & t-value & p-value & $95 \% \mathrm{Cl}$ \\
\hline Preimplantation & $62.2 \pm 22.0 \mathrm{mg}$ & & & \\
\hline Post 1-week & $42.2 \pm 20.7 \mathrm{mg}$ & 16.322 & $<0.001$ & $17.51-22.49$ \\
\hline Post 1-month & $26.2 \pm 15.7 \mathrm{mg}$ & 24.396 & $<0.001$ & $32.96-38.93$ \\
\hline Post 2-month & $60.3 \pm 25.7 \mathrm{mg}$ & 1.312 & 0.198 & $-1.03-4.82$ \\
\hline
\end{tabular}

Exclusion criteria were patients who could not tolerate puncture under local anesthesia, multiple organ failure, pancreatic cancer complicated with acute pancreatitis; coagulation dysfunction not improved after treatment; severe Diabetes, and the blood sugar could not be controlled below 16.7 $\mathrm{mmol} / \mathrm{L}$ after hypoglycemic treatment.

Clinical symptoms, vital signs, electrocardiogram, routine blood tests, clotting function, routine urine tests, liver function, renal function, blood electrolytes, blood glucose, blood amylase, urine amylase, cardiopulmonary function were observed or examined before CTRISI for each patient. Abdominal contrast enhanced CT scan was performed to determine the size, location and relation of tumor with adjacent structures. The number and distribution of 125 I seeds were determined by treatment planning system (TPS). The volume of the target area (planning target volume, PTV) was defined as the external expansion by one $\mathrm{cm}$ of the tumor target area, matched peripheral dose was 110-160 Gy. The patients were advised not to take anything orally for 12 hours prior to the procedure in order to avoid bowel injury during operation. During the operation, the appropriate puncture path and puncture point were selected by CT scan (Figure 1). Routine disinfection of the skin, laying sterile hole towel, with $2 \%$ lidocaine for local anesthesia. Then, under the guidance of CT, 125I seeds were implanted into the tumor at $0.5-1.0 \mathrm{~cm}$ intervals for interstitial radiotherapy. The dose of radiation was evaluated by $\mathrm{CT}$ after operation, and the cold zone of radiation was supplemented with implanted seeds.
Fasting was continued until $48 \mathrm{~h}$ after operation, while strengthening total parenteral nutrition (TPN), and hemostatic drugs were routinely used to control topical bleeding. Radiation protection was used in all clinical and nursing procedures.

Thirty seven patients were followed-up in outpatient department or by telephone at least 2-months. The changes in pain intensity before and after operation were observed. NRS was used to record the pain score: 0 was no pain, 10 was severe pain and unbearable, 1-3 was mild pain, 4-6 was moderate pain, and 7-9 was severe pain. The recording time was 1 week before and 1 week, 1 month and 2 months after treatment. Follow-up CT scanning was done 2 months after the first implantation to determine whether reimplantation was required based on tumor size and pain relief.

Statistical analysis was performed using Statistical Package for Social Sciences (SPSS), version 20.0 (IBM Corp., Armonk, NY, USA). Continuous variables were represented as range mean \pm standard deviation and numerical data was expressed as frequency with percentages. Repeated measures ANOVA and paired t-test were used for comparison between pre-and posttreatment groups. The $p$-values less than or equal to 0.05 were considered significant.

\section{RESULTS}

All patients achieved successful CTRISI as shown in repre-sentative Figures 1 and 2 . The average implantation of $125 \mathrm{I}$ seeds was $30.0 \pm 8.978(20-60)$, the average operation time was 
$55.649 \pm 11.434$ minutes (40-90), and the average hospitalisation time was $9.622 \pm 1.063$ days (8-12).

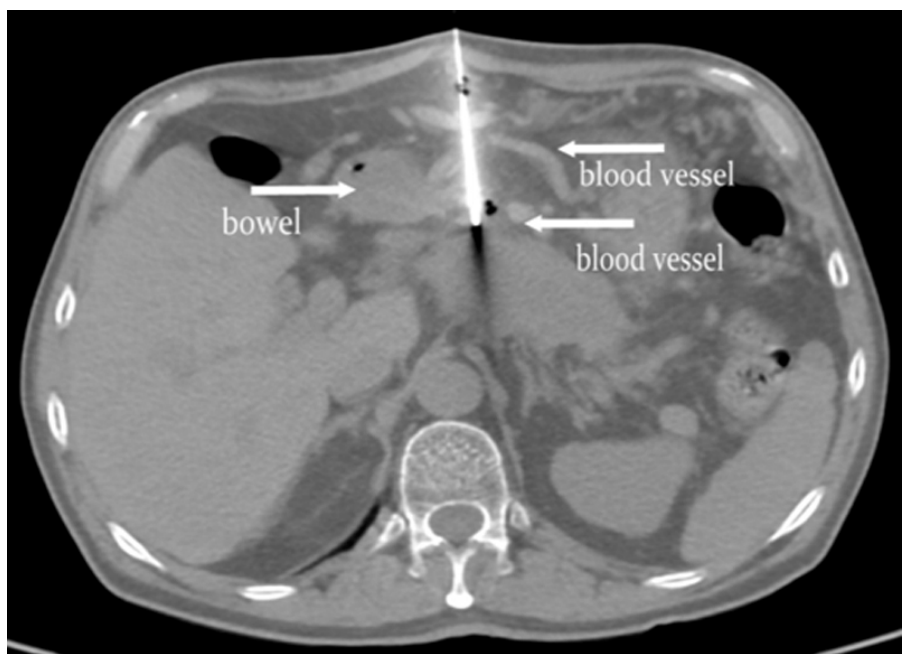

Figure 1: A 63-year-old male patient with advanced pancreatic cancer was given 60 pieces of 125 I seeds implanted once. During the front entry path operation, the puncture needle bypassed blood vessels and bowels without damage.

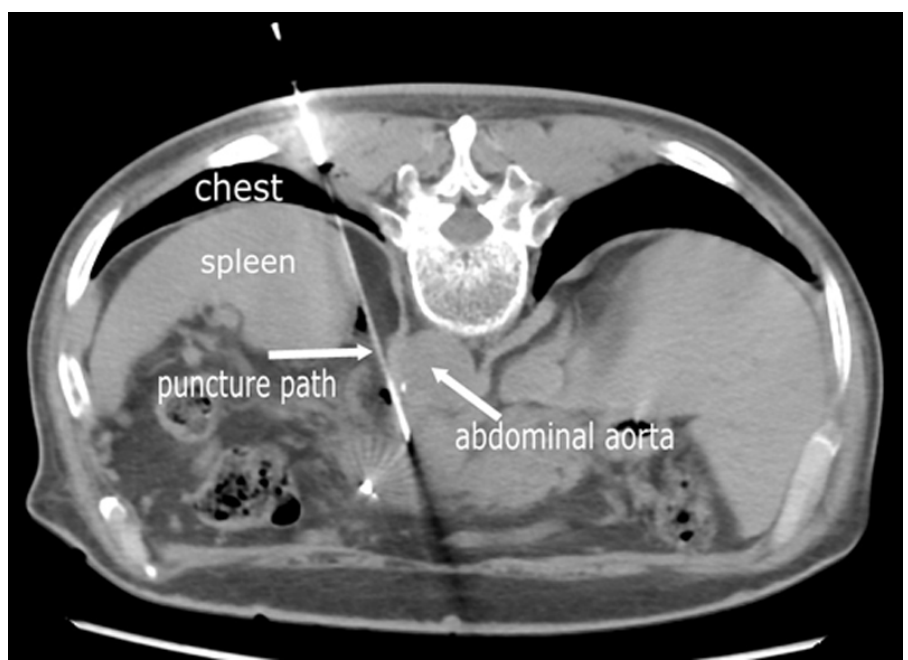

Figure 2: CT image of a patient with pancreatic cancer (male, 78-yearold). During the rear entry path operation, the patient was successfully implanted $125 \mathrm{I}$ seeds without hemorrhage and pneumothoraxunder accurate CT-guidance.

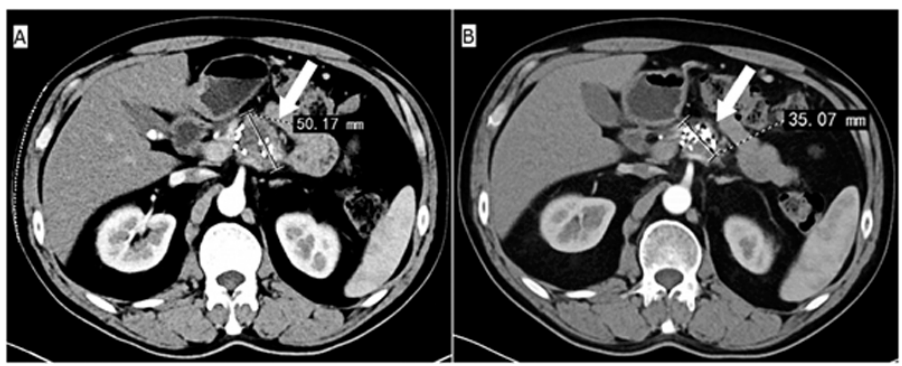

Figure 3: Representative images showing curative effect of $125 \mathrm{I}$ seeds implantation in a patient (male, 51-year-old) with pancreatic cancer pain. (A) The pancreatic cancer size on the day of treatment. (B) The pancreatic cancer size after one month of treatment.

Before CTRISI, 12 patients had mild pain, 24 patients had moderate pain, and one patient had severe pain. After 1 -week of the implantation, 1 patient had $100 \%$ pain relief, 23 patients had at least $50 \%$ pain relief, and the rest 13 patients had no response to the treatment. There was no pain in 1 case, mild pain in 34 cases, moderate pain in 2 cases and severe pain in none. Compared to the situation, after 1 -week, the no and mild pain $(94.6 \%, 35 / 37)$ were significantly higher than pre-operative scores $(32.4 \%, 12 / 37)$.

One month after operation, 2 patients had $100 \%$ pain relief, 20 patients had at least $50 \%$ pain relief, and the rest 15 patients had no response to the treatment. There were 2 cases without pain, 30 cases with mild pain, 5 cases with moderate pain and no case with severe pain. After one month, the incidence of no and mild pain $(86.5 \%, 32 / 37)$ was significantly higher than that before operation $(32.4 \%, 12 / 37)$. After 2 -month, 0 case of no pain, 21 cases of mild pain, 16 cases of moderate pain, 0 case of severe pain. Compared with preoperative, no and mild pain $(56.8 \%, 21 / 37)$ was also higher than preoperative $(32.4 \%$, $12 / 37)$. The changes in pain intensity before and after CTRISI are shown in Table I.

After 1-week, 1-month and 2-month of the CTRISI implantation, the NRS scores were significantly lower than preoperative scores $(p<0.05)$. The repeated measure ANOVA comparisons of NRS score before and after CTRISI are shown in Table II.

The frequency of pain relief after 1-week, 1-month and 2month of the implantation were $67.6 \%(25 / 37), 59.5 \%(22 / 37)$ and $27.0 \%$ (10/37), respectively.

The daily oral hydroxycodone dose after CTRISI 1-week and 1month were significantly lower than preimplantation doses $(p<0.001)$. However, there was no difference between the daily dose of hydroxycodone after 2 months and the preimplantation dose $(p=0.198)$. The changes in daily hydroxycodone dose before and after CTRISI are shown in Table III.

No patient experienced serious complications, such as uncontrolled bleeding, intestinal obstruction, pancreatic fistula, and abdominal infection. Among them, 5 patients experienced mild, transient side effects (increased serum amylase, reflex hypotension, and fervescence) that resolved within 1-3 days. After symptomatic treatment, they all improved quickly.

\section{DISCUSSION}

Pancreatic cancer is a highly malignant digestive system tumor with low surgical resection rate, with 5-year survival rates in the range of $8 \%-10 \%{ }^{8}$ Eighty to eighty five percent of patients with advanced pancreatic cancer have different degrees of cancer pain. ${ }^{9-12}$ Pancreatic cancer pain not only makes patients intolerable, greatly affects the quality of life, but also may lead to a series of negative emotions, thus affecting the treatment effect. Therefore, relieving pain and improving the quality of life of patients has become an important part of comprehensive treatment of pancreatic cancer.

In recent years, radioactive 125 I seed implantation radiotherapy has achieved significant clinical effect, especially in the treatment of cancer pain. ${ }^{13-15}$ A study shows that the cost of the percutaneous seed implantation is similar to that of conventional radiotherapy. ${ }^{16}$ Besides, once 125 I radioactive particles are implanted into tumor tissue, the effect of radiation lasts longer inside tumor tissue without injuring normal tissues. Percu- 
taneous particles implantation has many benefits, such as lower operation risk, shorter operative time, lesser blood loss, rapid postoperative recovery, and no abdominal scar. Jin et al. reported that the Visual Analogue Score (VAS) at 1-week and 1month after CTRISI were $1.73 \pm 1.91$ and $3.53 \pm 1.51$, respectively, which were significantly lower than $5.07 \pm 2.63$ before implantation. ${ }^{17}$ In other studies, the effective rate of pain relief (VAS $\leq 3)$ after CTRISI was $69.2 \%(9 / 13)$ and $87.5 \%(7 / 8) .{ }^{18} \mathrm{In}$ this study, after 1 -week, 1-month and 2 -month of the implantation, the NRS scores were $2.5 \pm 0.9,2.5 \pm 1.0$ and $3.4 \pm 0.9$, respectively, which were significantly lower than $4.0 \pm 1.6$ before implantation. Preimplantation compared with post 1week and 1-month, the $p$-values were less than 0.001 . The $p$ value was 0.005 between preimplantation and post 2-month.

As we know pancreatic cancer grows rapidly and reaches larger size and often causes pressure effects on surrounding tissues and nerve, leading to continual tissue swelling and apparent pain. ${ }^{19,20}$ When the tumor shrinks, pressure to the surrounding tissues will be gradually decreased, and it is completely understandable that pain relief is closely associated with the tumor control, as show in the Figure 3.

In this study, the daily oral dose of hydroxycodone was 62.2 $\pm 22.0 \mathrm{mg}$ before operation, and 1-week, 1-month and 2-month after operation were $42.2 \pm 20.7 \mathrm{mg}, 26.2 \pm 15.7 \mathrm{mg}$ and 60.3 $\pm 25.7 \mathrm{mg}$, respectively. One week and one month after implantation, the daily dose of hydroxycodone was significantly lower than that before implantation $(p<0.001)$, and the quality of life was significantly improved. However, with the develop-ment of tumor and the invasion of peripancreatic nerve, there was no difference between the daily dose of hydroxycodone after 2 months and the pre implantation dose $(p=0.198)$. Of course, it may also be that the repeated use of hydroxycodone produces tolerance and requires more hydroxycodone dosage to achieve analgesic effect.

CT guided $125 \mathrm{I}$ radioactive seeds implantation in the treatment of intractable pain of pancreatic cancer can not only effectively reduce tumor volume, relieve pain and reduce the amount of painkillers, but also greatly reduce the adverse reactions compared with traditional surgery. In our study, there were no patients with uncontrolled bleeding, intestinal obstruction, pancreatic fistula, abdominal infection and other serious complications. Only 5 patients had mild, transient side effects (increased serum amylase, reflex hypotension and fever) within 1-3 days. After symptomatic treatment, they all improved quickly.

CTRISI technology is a combination of surgical techniques and advantages of radiation therapy. Although, it may not completely resolve the pain caused by cancer, but it can reduce the NRS score quickly, reduce the amount of painkillers obviously and improve the quality of life in the majority of patients with pancreatic cancer pain.

\section{CONCLUSION}

CTRISI technology is minimally invasive, safe, quick and efficient in the treatment of unresectable pancreatic cancer patients with intractable pain. It can effectively relieve pain, reduce NRS score, reduce the dosage of painkillers and improve the quality of life of patients.

\section{ACKNOWLEDGEMENT:}

This project was funded/supported by joint fund project of Hubei Provincial Health Committee (WJ2019H510) and Yichang Social Science Fund (ysk17kt231).

\section{ETHICAL APPROVAL:}

This study had prior formal approval of hospital's Ethical and Research Committee of First Affiliated Hospital of Zhengzhou University, China.

\section{PATIENTS' CONSENT:}

Informed consent was obtained from subjects.

\section{CONFLICT OF INTEREST:}

Authors declared no conflict of interest.

\section{AUTHORS' CONTRIBUTION:}

TF: Conception and design of the work; acquisition, analysis, interpertation of data.

JYZ: Drafted and reviewed the paper, advices and final approval.

\section{REFERENCES}

1. Wu J, Liu J, Wei XQ, Yu Q, Niu XH, Tang SH, et al. A featurebased analysis identifies COL1A2 as a regulator in pancreatic cancer. J Enzyme Inhib Med Chem 2019; 34:420-8.

2. Gurusamy KS, Kumar S, Davidson BR, Fusai G. Resection versus other treatments for locally advanced pancreatic cancer. Cochrane Database Syst Rev 2014; 2:CD010244.

3. Tseng CM, Huang SP, Liao WC, Chiang CJ, Yang YW, Chang CY, et al. Incidence and mortality of pancreatic cancer on a rapid rise in Taiwan, 1999-2012. Cancer Epidemiol 2017; 49:75-84.

4. Ilic M, Ilic I. Epidemiology of pancreatic cancer. World J Gastroenterol 2016; 22:694-705.

5. McDonald PC, Chafe SC, Brown WS, Saberi S, Swayampakula $\mathrm{M}$, Venkateswaran $\mathrm{G}$, et al. Regulation of pH by Carbonic Anhydrase 9 Mediates Survival of Pancreatic Cancer Cells With Activated KRAS in Response to Hypoxia. Gastroenterology 2019; 157:823-37.

6. Gasparini G, Pellegatta M, Crippa S, Lena MS, Belfiori G, Doglioni $C$, et al. Nerves and Pancreatic Cancer: New Insights into a Dangerous Relationship. Cancers (Basel) 2019; 11.

7. Dobosz $Ł$, Kaczor M, Stefaniak TJ. Pain in pancreatic cancer: review of medical and surgical remedies. ANZ J Surg 2016; 86:756-61.

8. Shaib WL, Jones JS, Goodman M, Sarmiento JM, Maithel SK, Cardona $\mathrm{K}$, et al. Evaluation of treatment patterns and survival outcomes in elderly pancreatic cancer patients: A surveillance, epidemiology, and end results-medicare analysis. Oncologist 2018; 23:704-11.

9. Lou S. Endoscopic Ultrasound-guided Celiac Plexus Neurolysis to Alleviate Intractable Pain Caused by Advanced Pancreatic Cancer . Surg Laparosc Endosc Percutan Tech 2019; 1-4.

10. Oh TK, Do SH, Yoon YS, Song IA. Association between opioid use and survival time in patients with unresectable pancreatic cancer: 10 years of clinical experience. Pancreas 2018; 47: $837-42$.

11. Koulouris Al, Banim P, Hart AR. Pain in patients with pancreatic cancer: Prevalence, mechanisms, management and future 
developments. Dig Dis Sci 2017; 62:861-70.

12. Woo SM, Song MK, Lee M, Joo J, Kim DH, Kim JH, et al. Effect of Early Management on Pain and Depression in Patients with Pancreatobiliary Cancer: A Randomized Clinical Trial . Cancers (Basel) 2019; 11:1-6.

13. Liu K, Ji B, Zhang W, Liu S, Wang Y, Liu Y. Comparison of iodine-125 seed implantation and pancreaticoduodenectomy in the treatment of pancreatic cancer. Int J Med Sci 2014; 11:893-6

14. Hu Y, Qi E, Liu F, Lu Y, Tan S, Sun Y, et al. The application of a three-dimensional visualized seed planning and navigation system in 125 I seed implantation for pancreatic cancer. Onco Targets Ther 2018; 11:619-27.

15. Gai B, Zhang F. Chinese expert consensus on radioactive $125 \mathrm{I}$ seeds interstitial implantation brachytherapy for pancreatic cancer.J Cancer Res Ther 2018; 14:1455-62.

16. Li Q, Tian Y, Yang D, Liang $Y$, Cheng $X$, Gai B. Permanent lodine-125 seed implantation for the treatment of nonresectable retroperitoneal malignant tumors. Technol Cancer Res Treat 2019; 18:1-8.

17. Jin Z, Du Y, Li Z, Jiang Y, Chen J, Liu Y. Endoscopic ultrasonography-guided interstitial implantation of lodine 125-seeds combined with chemotherapy in the treatment of unresectable pancreatic carcinoma: a prospective pilot study. Endoscopy 2008; 40:314-20.

18. Zhongmin W, Yu L, Fenju L, Kemin C, Gang H. Clinical efficacy of CT-guided iodine-125 seed implantation therapy in patients with advanced pancreatic cancer. Eur Radiol 2010; 20: 1786-91.

19. Bapat AA, Hostetter G, Von Hoff DD. Perineural invasion and associated pain in pancreatic cancer. Nat Rev Cancer 2011; 11:695-707.

20. Moore JC, Adler DG. Celiac plexus neurolysis for pain relief in pancreatic cancer. J Support Oncol 2009; 7:83-7. 